\title{
LACTOFERRIN FOR PREVENTION OF SEPSIS AND NECROTIZING ENTEROCOLITIS
}

\author{
P. Manzoni ${ }^{1}$, M. Mostert ${ }^{2}$, M. Stronati ${ }^{3}$, D. Farina ${ }^{1}$ \\ ${ }^{I}$ Neonatology and NICU, Ospedale Infantile Regina Margherita Sant'Anna, ${ }^{2}$ University of Torino, Turin, \\ ${ }^{3}$ IRCCS Policlinico S. Matteo, Pavia, Italy
}

Neonatal sepsis cause severe morbidity and mortality. Neonates in NICU feature many specific risk factors for bacterial and fungal sepsis. Loss of gut commensals such as Bifidobacteria/Lactobacilli, as occurs with prolonged antibiotic treatments, delayed enteral feeding, or nursing in incubators, translates into proliferation of pathogenic microflora and abnormal gut colonization,

Prompt diagnosis and effective treatment do not protect septic neonates from the risk of late neurodevelopmental impairment in the survivors, thus specific prevention is mandatory in these particular settings.

In this view, improving Neonatal Management is a key step. This include promoting Breastfeeding and Hygiene measures, cautious CVC management, enhancing Enteric microbiota composition with Probiotics, and medical stewardship concerning H2-blockers/steroids restrictions.

A new approach towards reduction of sepsis and NEC might involve the use of bioactive substances with known anti-infective properties. Lactoferrin is a mammalian milk glycoprotein involved in innate immune host defences, and can reduce the incidence of late-onset sepsis in VLBW infants (MANZONI JAMA 2009) and of NEC in animal models. The bovine isoform is nearly homologous to the human one. Lactoferrin targets all pathogens, has bifidogenic properties, and enhance maturation of the nascent gut.

In a recent RCT, bovine LF produced a $65 \%$ decrease in any-cause LOS, and significant decrease in both surgical and low-stages NEC (MANZONI PAS 2011).

As no adverse effects or intolerances to treatment have been reported todate, the role of LF in the management of infections and NEC in NICU looks very promising and worthy of future, larger-sized trials to confirm these findings. 